\title{
Phase stability of Au-Li binary systems studied using neural network potential
}

\author{
Koji Shimizu $\odot,{ }^{1},{ }^{*}$ Elvis F. Arguelles $\odot,{ }^{1}$ Wenwen $\mathrm{Li},{ }^{2}$ Yasunobu Ando $\odot,{ }^{2}$ Emi Minamitani $\odot,{ }^{3}$ and Satoshi Watanabe $\odot^{1, \dagger}$ \\ ${ }^{1}$ Department of Materials Engineering, The University of Tokyo, 7-3-1 Hongo, Bunkyo-ku, Tokyo 113-8656, Japan \\ ${ }^{2}$ Research Center for Computational Design of Advanced Functional Materials, National Institute of Advanced Industrial Science \\ and Technology (AIST), 1-1-1 Umezono, Tsukuba, Ibaraki 305-8568, Japan \\ ${ }^{3}$ Institute for Molecular Science, 38 Nishigo-Naka, Myodaiji, Okazaki 444-8585, Japan
}

(Received 11 July 2020; revised 22 February 2021; accepted 9 March 2021; published 22 March 2021)

\begin{abstract}
The miscibility of Au and Li exhibits a potential application as an adhesion layer and electrode material in secondary batteries. Here, to explore alloying properties, we constructed a neural network potential (NNP) of AuLi binary systems based on density functional theory (DFT) calculations. To accelerate construction of NNPs, we proposed an efficient and inexpensive method of structural dataset generation. The predictions by the constructed NNP on lattice parameters and phonon properties agree well with those obtained by DFT calculations. We also investigated the mixing energy of $\mathrm{Au}_{1-x} \mathrm{Li}_{x}$ with fine composition grids, showing excellent agreement with DFT verifications. We found the existence of various compositions with structures on and slightly above the convex hull, which can explain the lack of consensus on the $\mathrm{Au}-\mathrm{Li}$ stable phases in previous studies. Moreover, we found other stable phases, namely $\mathrm{Au}_{0.45} \mathrm{Li}_{0.55}, \mathrm{Au}_{0.389} \mathrm{Li}_{0.611}$, and $\mathrm{Au}_{0.357} \mathrm{Li}_{0.643}$. Finally, we examined the alloying process starting from the phase separated structure to the complete mixing phase. We found that when multiple adjacent $\mathrm{Au}$ atoms dissolved into $\mathrm{Li}$, the alloying of the entire $\mathrm{Au} / \mathrm{Li}$ interface started from the dissolved region. This paper demonstrates the applicability of NNPs toward miscible phases, and it provides an understanding of the alloying mechanism.
\end{abstract}

DOI: 10.1103/PhysRevB.103.094112

\section{INTRODUCTION}

The Au-Li binary system is known to exhibit various alloy phases over a wide range of compositional ratios [1-4]. This remarkable alloy miscibility has recently been attracting attention due to its potential applications, most specifically in next-generation energy storage and electronic devices, among others. In Li-ion batteries (LIBs), Au is shown to form high-Li-concentration stable alloy phases at low voltages in addition to the low alloying/dealloying potential. This implies that $\mathrm{Au}$ is a promising candidate for an anode electrode material in LIBs [5]. Moreover, thin-layer Au inserted at the interface of the $\mathrm{Li}$-anode and a solid electrolyte can suppress void formations, resulting in enhanced cyclability and a reduction of interface resistance [6,7]. In novel nonvolatile memory devices, the amount of $\mathrm{Li}$ at the interface of the $\mathrm{Au}$ cathode and the $\mathrm{Li}_{3} \mathrm{PO}_{4}$ solid electrolyte controls switching behavior $[8,9]$. These aspects highlight the significance of atomic scalebased studies of Au-Li structural properties and to what extent the alloying proceeds in materials design and the development of electrochemical devices.

Experiments using thermal analysis and x-ray measurement report the observed $\mathrm{Au}-\mathrm{Li}$ phases to be $\alpha \quad(17-40 \quad$ at. $\% \quad \mathrm{Li}), \quad \mathrm{Au}_{5} \mathrm{Li}_{4}, \quad \beta \quad(47-56 \quad$ at. $\%$ $\mathrm{Li}), \delta(62.5-65.5$ at. $\% \mathrm{Li}), \mathrm{AuLi}_{3}$, and $\mathrm{Au}_{4} \mathrm{Li}_{15}$

\footnotetext{
*shimizu@cello.t.u-tokyo.ac.jp

${ }^{\dagger}$ watanabe@cello.t.u-tokyo.ac.jp
}

[1]. Electrochemical measurements and theoretical calculations identify the $\mathrm{AuLi}_{3}$ and $\mathrm{Au}_{2} \mathrm{Li}$ phases as the stable alloy phases, and $\mathrm{Au}_{3} \mathrm{Li}_{5}, \mathrm{Au}_{2} \mathrm{Li}_{3}$, and $\mathrm{Au}_{5} \mathrm{Li}_{3}$ as the metastable phases [2]. Another theoretical work based on density functional theory (DFT) calculations presents $\mathrm{Au}_{3} \mathrm{Li}$, $\mathrm{AuLi}, \mathrm{AuLi}_{2}$, and $\mathrm{AuLi}_{3}$ as stable phases at $0 \mathrm{GPa}$ [4]. The available stable phases stored in the Materials Project (MP) [10,11] database are $\mathrm{Au}_{3} \mathrm{Li}, \mathrm{AuLi}, \mathrm{AuLi}_{3}$, and $\mathrm{Au}_{4} \mathrm{Li}_{15}$. Discrepancies among the previous studies seen above arise from the intrinsic complexity of miscible alloy materials. Furthermore, high computational costs loaded in a theoretical investigation over a wide compositional space contribute to the lack of consensus on the stable Au-Li phases.

Conventional theoretical studies on alloying properties of other materials also employ DFT calculations to obtain the mixing energies of various compositions and to draw phase diagrams [12-14]. However, in most cases, research studies focus on a few stable phases due to the aforementioned formidable computational costs of a comprehensive search over a wide range of compositions at the $a b$ initio level. Alternatively, classical molecular dynamics (MD) simulations using empirical interatomic potentials are able to take into consideration various computational conditions; however, the accuracy of this approach is limited by the quality of the empirical interatomic potentials, which is often insufficient. In recent years, machine learning (ML) interatomic potentials, e.g., neural network potential (NNP) [15], Gaussian approximation potential (GAP) [16], and spectral neighbor analysis potential (SNAP) [17], have been gaining much attention be- 
cause of their lower computational costs by several order of magnitude while achieving comparable accuracy with DFT calculations.

In particular, ML potentials have been successfully applied to binary and ternary alloy systems [18-22,24-26]. The NNP of GeTe provides structural quantities of liquid, crystalline, and amorphous phases comparable with DFT calculations [18]. The nanoalloy properties (e.g., structures and compositions) of $\mathrm{Cu}-\mathrm{Au}$ [19] and $\mathrm{Ag}-\mathrm{Au}$ [20] are well predicted by the NNPs. The NNPs of the Cu-Pd, Cu-Ag, Pd-Ag, and $\mathrm{Cu}-\mathrm{Pd}-\mathrm{Ag}$ systems reproduce defect and formation energies and phonon properties well in comparison to DFT calculations [21], wherein an efficient way of constructing multielement NNPs is proposed, the so-called stratified NN. The NNPs also have been applied to Al-Mg-Si alloys [22], $\mathrm{Li}-\mathrm{Si}$ alloys [23,24], and Pd-Si alloys [25], where all NNPs accurately predict physical properties comparable to DFT results. Moreover, the Ni-Mo interatomic potential constructed using SNAP has demonstrated that the predicted phase diagram agrees well with the experiment, as well as the DFT-level prediction of various physical properties [26]. The ML potentials have also been applied to simulate $\mathrm{Li}$-ion diffusion in amorphous$\mathrm{Li}_{3} \mathrm{PO}_{4}$ [27] and in $\mathrm{Li}_{10} \mathrm{GeP}_{2} \mathrm{~S}_{12}$ and $\mathrm{Li}_{7} \mathrm{La}_{3} \mathrm{Zr}_{2} \mathrm{O}_{12}$ [28], and to simulate the Li intercalations in carbon [29] anodes. These potentials were able to accurately reproduce their corresponding DFT quantities and exhibited better performance in comparison to empirical potentials.

As far as our literature search is concerned, ML potentialbased investigations on the Au-Li binary system have not yet been reported. Given their ability to reliably reproduce the different $a b$ initio quantities at lower computational cost, ML potentials may be able to offer some resolutions on the abovementioned discrepancies in this system. Despite its successes in predicting alloy properties, ML potentials are not without challenges. One such challenge is the proper generation of structural datasets needed for training the NNP, which is often achieved through ab initio molecular dynamics (AIMD) simulations. The reliability of NNP depends strongly on the randomness of the structural features of the dataset, which in turn requires long simulation runs. To the best of our knowledge, a robust method of generating and preparing datasets for NNP training has not yet been developed.

In the present study, we constructed an NNP for the Au-Li binary system in an efficient and inexpensive way of structural dataset generation, which we used to investigate its alloying properties. The accuracy of the NNP was corroborated by calculating the equation of state and phonon dispersions of some representative $\mathrm{Au}-\mathrm{Li}$ alloy structures and comparing them with DFT results. From the mixing energies of $\mathrm{Au}_{1-x} \mathrm{Li}_{x}$, we found that there were several structures at various alloy compositions that lay on or slightly above the convex hull, which could explain the discrepancies among the previously reported alloy phases. We also found additional stable $\mathrm{Au}-\mathrm{Li}$ alloy phases. In conjunction with these findings, we present an efficient method of structural dataset generation for NNP training, which allows an inexpensive construction of accurate NNPs.

This paper is organized as follows. Section II describes the computational conditions of DFT and NNP, and the procedure to construct the NNP. In Sec. III, we present the results

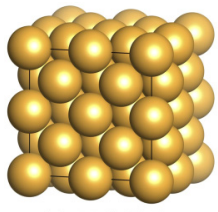

(a) FCC Au

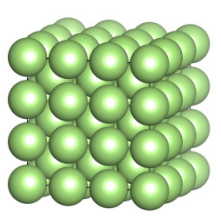

(c) $\mathrm{BCC} \mathrm{Li}$

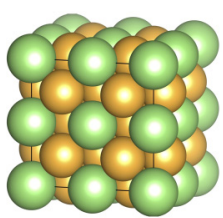

(e) $\mathrm{Au}_{3} \mathrm{Li}$

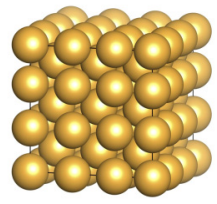

(b) $\mathrm{BCC} \mathrm{Au}$

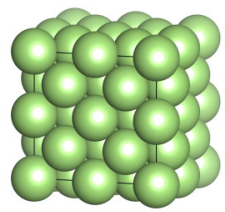

(d) FCC Li

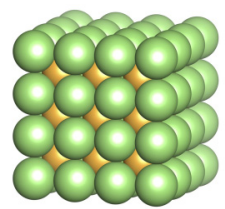

(f) $\mathrm{AuLi}$

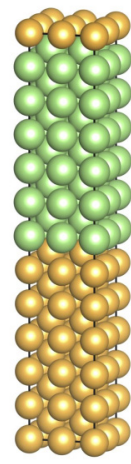

(h) BCC AuLi

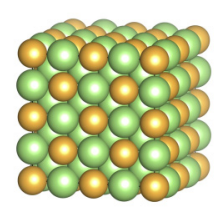

(g) $\mathrm{AuLi}_{3}$
FIG. 1. Base structures of (a,b) fcc and bcc Au, (c,d) bcc and fcc $\mathrm{Li}$, (e) fcc $\mathrm{Au}_{3} \mathrm{Li}$, (f) bcc AuLi, (g) rocksalt $\mathrm{AuLi}_{3}$, and (h,i) bcc and fcc $\mathrm{Au}_{0.5} \mathrm{Li}_{0.5}$ superlattices, used in the training dataset. Structures are visualized using the VESTA package [30].

of predicted physical properties using the constructed NNP. The stable phase search and alloying process of the phase separated structure are also provided in Sec. III. Finally, conclusions are presented in Sec. IV.

\section{METHODOLOGY}

\section{A. DFT calculations}

We first performed DFT-based AIMD calculations using the following structures to generate a temporal structural dataset for NNP construction: face-centered-cubic (fcc) Au, body-centered-cubic (bcc) $\mathrm{Au}$, bcc $\mathrm{Li}$, fcc $\mathrm{Li}$, ordered fcc $\mathrm{Au}_{3} \mathrm{Li}$ (32 atoms/supercell), bcc AuLi (54 atoms/supercell), and rocksalt $\mathrm{AuLi}_{3}$ (128 atoms/supercell), as well as $\mathrm{Au} / \mathrm{Li}$ superlattices of bcc $\mathrm{Au}_{0.5} \mathrm{Li}_{0.5}$ (96 atoms/supercell) and fcc $\mathrm{Au}_{0.5} \mathrm{Li}_{0.5}$ (160 atoms/supercell). The supercells of these structures are shown in Fig. 1 schematically.

We carried out constant temperature ( $N V T$-ensemble) AIMD simulations with $1 \mathrm{fs}$ time steps for $1 \mathrm{ps}$. The initial temperature was set to $300 \mathrm{~K}$ and linearly increased up to $2000 \mathrm{~K}$ (at $1.7 \mathrm{~K} / \mathrm{fs}$ ) to obtain largely random structural features. Considering the high computational costs of AIMD calculations, further structural generations were conducted using NNP-based MD simulations, and subsequent static DFT calculations were performed for the extracted structures.

For DFT calculations, we used the generalized gradient approximation with the Perdew-Burke-Ernzerhof functional [31], the plane-wave basis set (500 eV cutoff energy), and the projector augmented wave method [32]. Brillouin zone integration was performed using the sampling technique of Monkhorst and Pack $(5 \times 5 \times 5$ and $5 \times 5 \times 1$ sampling meshes for cubic cells and rectangular cells, respectively) [33]. We used Vienna Ab initio Simulation Package (VASP) software $[34,35]$ for all the DFT calculations. 


\section{B. NNP construction}

We adopted a high-dimensional NNP [15] to describe the interatomic potential of $\mathrm{Au}$ and $\mathrm{Li}$ atoms. In this scheme, the information of atomic structures is encoded as the values of symmetry functions (SFs) and is related to the energy contribution of each atom, $E_{i}$, via the neural network. The total energy of the system is then described by the sum over the energy contributions of the respective atoms $(E=$ $\left.\sum_{i} E_{i}\right)$. Subsequently, the forces along the atomic coordinates $\alpha=x, y, z$ can be expressed as $F_{\alpha_{i}}=-\partial E / \partial \alpha_{i}=$ $-\sum_{v} \partial E / \partial G_{v} \times \partial G_{v} / \partial \alpha_{i}$, where $v$ labels the type of SFs. We used the radial $\left(G_{2}\right)$ and angular $\left(G_{3}\right)$ SFs in the following forms:

$$
\begin{gathered}
G_{2}^{i}=\sum_{j \neq i} \mathrm{e}^{-\eta\left(R_{i j}-R_{s}\right)^{2}} f_{\mathrm{c}}\left(R_{i j}\right), \\
G_{3}^{i}=2^{1-\zeta} \sum_{j \neq i} \sum_{k \neq i, j}\left(1+\lambda \cos \theta_{i j k}\right)^{\zeta} \mathrm{e}^{-\eta\left(R_{i j}^{2}+R_{i k}^{2}\right)} \\
\times f_{\mathrm{c}}\left(R_{i j}\right) f_{\mathrm{c}}\left(R_{i k}\right),
\end{gathered}
$$

where $\eta$ and $\zeta$ are the width parameters; $\lambda$ and $R_{s}$ determine the peak positions; $R_{i j}$ and $R_{i k}$ are the atomic distances of atom $i$ with $j$ and $k$, respectively; and $\theta_{i j k}$ is the angle consisting of atoms $i, j$, and $k$ at the vertex $i . f_{\mathrm{c}}$ is the cutoff function given by

$$
f_{\mathrm{c}}\left(R_{i j}\right)= \begin{cases}\frac{\left[\cos \left(\frac{\pi R_{i j}}{R_{\mathrm{c}}}\right)+1\right]}{2} & \left(R_{i j} \leqslant R_{\mathrm{c}}\right), \\ 0 & \left(R_{i j}>R_{\mathrm{c}}\right),\end{cases}
$$

where $R_{\mathrm{c}}$ is the cutoff distance.

The set of hyperparameters used in the present study are shown in Sec. S1 and Table S1 in the supplemental material [36]. We used 6 and 18 kinds of radial and angular SFs, respectively, for each elemental combination. The combinations for $\mathrm{Au}$ atoms include $\mathrm{Au}-\{\mathrm{Au}, \mathrm{Li}\}$ and $\mathrm{Au}-\{\mathrm{Au}-\mathrm{Au}, \mathrm{Au}-\mathrm{Li}$, $\mathrm{Li}-\mathrm{Li}\}$ for radial and angular SFs, respectively. Similarly, the combinations of SFs for $\mathrm{Li}$ atoms include $\mathrm{Li}-\{\mathrm{Au}, \mathrm{Li}\}$ and $\mathrm{Li}-\{\mathrm{Au}-\mathrm{Au}, \mathrm{Au}-\mathrm{Li}, \mathrm{Li}-\mathrm{Li}\}$. Thus, the total number of input nodes (i.e., values of SFs) is $2 \times 6+3 \times 18=66$ per atom. The SF values were normalized within the range of $[-1: 1]$ prior to passing through the NN.

Based on the total energies and atomic forces obtained by the DFT calculations, we optimized the weight parameters using the limited-memory Broyden-Fletcher-Goldfarb-Shanno (1-BFGS) algorithm [37] with the following loss function:

$$
\begin{aligned}
\Gamma(\mathbf{w})= & \alpha \sum_{i=1}^{N_{\text {train }}}\left(E_{i}^{\mathrm{NNP}}-E_{i}^{\mathrm{DFT}}\right)^{2} \\
& +\beta \sum_{i=1}^{N_{\text {train }}}\left\{\sum_{j=1}^{3 n_{i}}\left(F_{j}^{i, \mathrm{NNP}}-F_{j}^{i, \mathrm{DFT}}\right)^{2}\right\},
\end{aligned}
$$

where $\mathbf{w}$ is the weight parameter vector. $\alpha$ and $\beta$ determine the contribution ratio of energies and atomic forces into the loss function. In the beginning of the NNP training, we evaluated 50 different, randomly assigned weight parameter sets, in which each weight value was chosen within the range of [-1:1]. Afterward, we started 1-BFGS training with the smallest error weight set using energy differences, i.e., $\alpha=$

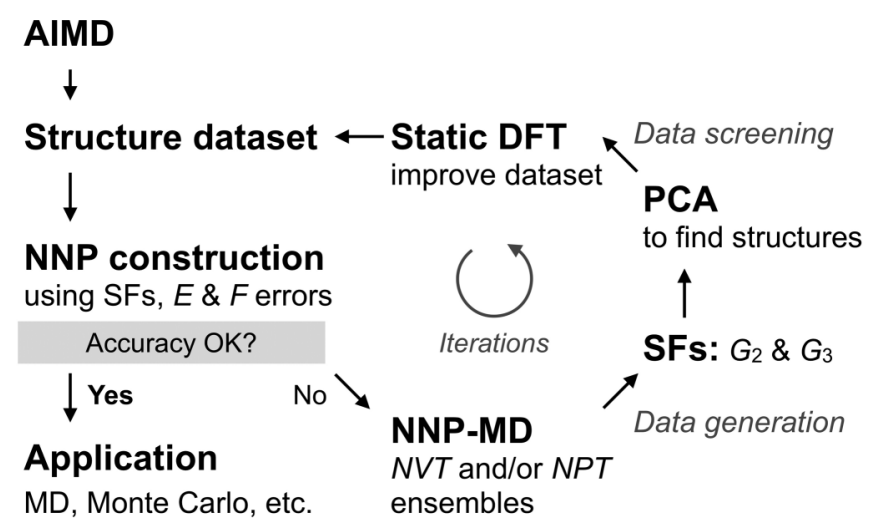

FIG. 2. A schematic description of the workflow of NNP construction and dataset generation.

1.0 and $\beta=0$. Subsequently, we continued the training using both energy and atomic force differences using the setting $\alpha=0.5$ and $\beta=0.5$.

\section{Structural dataset generation}

In this section, we will explain the efficient method of NNP construction and structural dataset generation that we developed for the present study. Figure 2 shows the workflow of our NNP construction. First, we constructed a tentative NNP using the structures obtained by the AIMD calculations (please refer to Sec. II A for the computational conditions). Although this tentative NNP is usually less accurate and insufficient for practical use, it was enough to be used for generating additional structures that were added to the training dataset. To obtain these additional structures, we coupled our NNP with Large-scale Atomic/Molecular Massively Parallel Simulator (LAMMPS) software [38,39] using our homemade interface and carried out MD calculations for the structures shown in Fig. 1. In the present study, we performed 10-100 ps NNP-MD with a 1 fs time step using both $N V T$ and NPT ensembles. The temperatures were set from 300 to $2100 \mathrm{~K}$ at $300 \mathrm{~K}$ intervals for the $\mathrm{Au}$ case, from 300 to $600 \mathrm{~K}$ at $100 \mathrm{~K}$ intervals for the $\mathrm{Li}$ case, and from 300 to $1200 \mathrm{~K}$ at $100 \mathrm{~K}$ intervals for the $\mathrm{Au}-\mathrm{Li}$ alloy cases. The pressures were set in the range of 0-1 GPa. The maximum temperatures were chosen based on being several hundreds of Kelvin higher than the materials' melting temperatures. The initial lattice parameters were chosen as the optimal values obtained by DFT. It is worth noting that rough NNP-MD simulations are often terminated by numerical errors due to structure collapses as mentioned in Ref. [40,41], wherein the training technique that can construct accurate and numerically stable NNPs was developed.

To select only those structures that have different structural features from the ones already included in the collected dataset, we performed principal component analysis (PCA) based on the SFs of each structure, where $66 \times N$ dimensional (where $N$ denotes number of atoms/structure) local atomic/structural features were reduced to twodimensional values, i.e., first and second principal components (PC1 and PC2). Note that the SF-based PCAs were previously performed in Refs. [24,42]. Having similar PC 

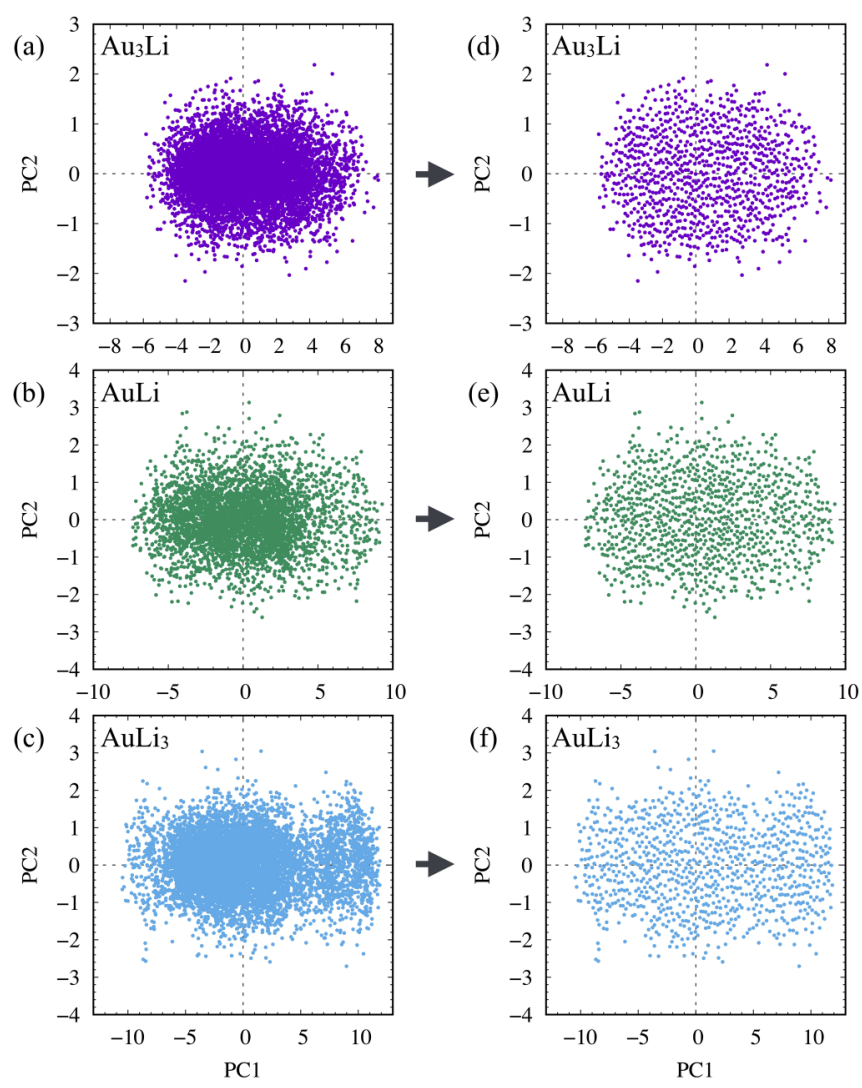

FIG. 3. Principal component plots of (left) a large number of structures generated by NNP-MD simulations, and (right) a reduced number of structures. (a,d) $\mathrm{Au}_{3} \mathrm{Li}$, (b,e) $\mathrm{AuLi}$, and (c,f) $\mathrm{AuLi}_{3}$ are shown as examples.

values means that those structures have similar structural features. In fact, structures resembling each other can be found frequently in MD trajectories. We calculated SFs for $1 \%$ of structures extracted from the NNP-MD trajectories. Then, we chose a specific number of structures by calculating the distances of neighboring points so that the selected PC values were well-scattered (please refer to Sec. S2 in the supplemental material for more details [36]). Figures 3(a)-3(c) show the PC plots of many of the structures of $\mathrm{Au}_{3} \mathrm{Li}, \mathrm{AuLi}$, and $\mathrm{AuLi}_{3}$, respectively. The plots of the extracted structures are shown in Figs. 3(d)-3(f). The well-scattered plots in all the cases presented suggest that the dataset contained various structures and atomic features (please also refer to Fig. S2 in the supplemental material for the resulting PC plots [36]). As an example, Fig. 4 shows the structures corresponding to the maximum and minimum PC values in rectangular fcc $\mathrm{Au}_{0.5} \mathrm{Li}_{0.5}$. The maximum PC1 corresponds to the phaseseparated structure. Conversely, at the minimum PC1 value, the $\mathrm{Au}$ and $\mathrm{Li}$ atoms were completely mixed. Both the maximum and minimum PC2 show the partial mixture of $\mathrm{Au}$ and $\mathrm{Li}$ atoms, which is reasonable because their $\mathrm{PC} 1$ values were located near the origin. The structures were more disordered as the PC2 value decreased, although the difference is rather ambiguous compared to PC1 cases. After PCA, we subsequently performed static DFT calculations for the new structures to obtain the total energies and atomic forces. These were then used to update our dataset and retrain our NNP.
FCC AuLi

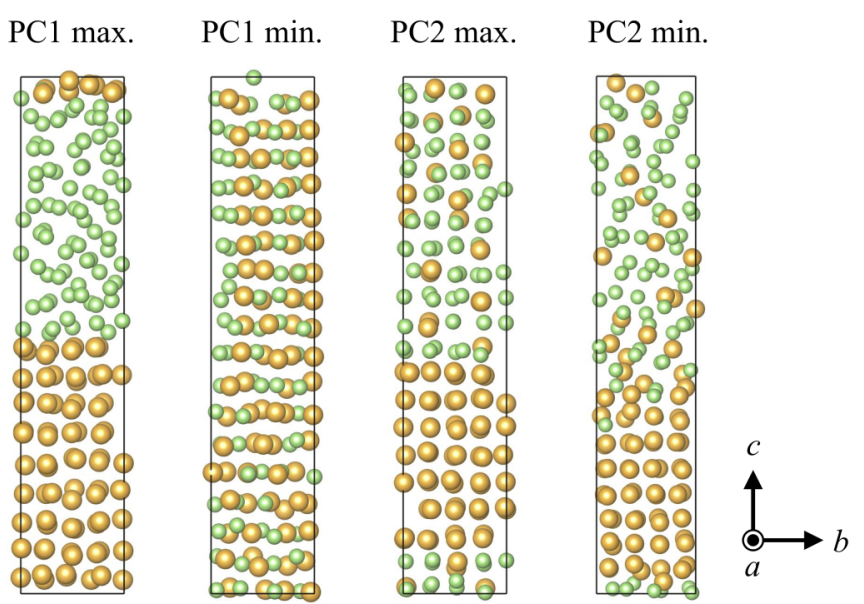

FIG. 4. Structures of rectangular fcc $\mathrm{Au}_{0.5} \mathrm{Li}_{0.5}$ at the boundaries of principal component (PC) values (cf. Fig. 3).

We performed this cycle iteratively until we could achieve an accurate NNP.

We generated 8696 structures in total, including $1973 \mathrm{Au}$, $1900 \mathrm{Li}, 949 \mathrm{Au}_{3} \mathrm{Li}, 992 \mathrm{AuLi}, 929 \mathrm{AuLi}_{3}, 971$ rectangular bcc $\mathrm{Au}_{0.5} \mathrm{Li}_{0.5}$, and 982 rectangular fcc $\mathrm{Au}_{0.5} \mathrm{Li}_{0.5}$ structures. However, we found that at low-Au-concentration alloys, the calculated mixing energies using the NNP seriously deviated from the DFT verifications. Therefore, we further added $589 \mathrm{Au}$ dilute alloy structures, i.e., $\mathrm{Au}_{1} \mathrm{Li}_{31}, \mathrm{Au}_{1} \mathrm{Li}_{53}$, and $\mathrm{Au}_{1} \mathrm{Li}_{127}$, obtained by the procedure explained above. Finally, our dataset containing 9285 structures covered the entire AuLi space, suggesting the versatility of the constructed NNP.

We note that Artrith and Behler proposed a method to find structures worth adding to the dataset in light of structural diversity using NNPs [43]. In their method, NNPs with different network architectures constructed by the same dataset are used to predict energies for a large number of structures (generated, for instance, by NNP-MD). A large difference in predicted energies of the same structure among NNPs indicates that its atomic features are missing in that dataset. In addition, Li and Ando applied this scheme to the on-the-fly sampling technique [44]. Compared with these previous methods, the present approach needed to construct only a single NNP. The lower computational costs for the training process would be the advantage against the above methods. Recently, Sivaraman et al., proposed another active learning approach of ML interatomic potential constructions, where the clustering of structures based on the pairwise root-mean-square deviations of atomic positions was used to detect structural similarities [45].

\section{RESULTS AND DISCUSSION}

\section{A. Au-Li binary system NNP}

Using the 9285 structures mentioned in the previous section, we constructed NNPs for the Au-Li binary system. We examined cutoff distances of 5,7 , and $9 \AA$ with the NNs consisting of 2 hidden layers and 10 or 20 nodes per hidden layer. The obtained root-mean-square-error (RMSE) values 

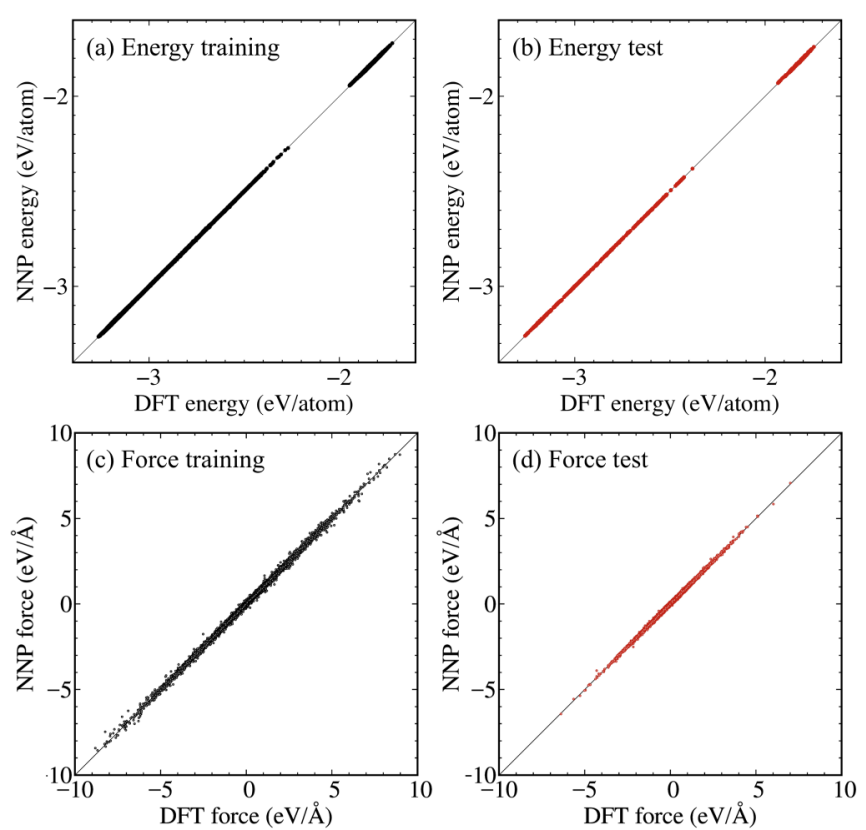

FIG. 5. Comparison between DFT and NNP on the total energies of (a) training and (b) test sets. Parts (c) and (d) show the comparison of the atomic forces for training and test sets, respectively.

are summarized in Sec. S1 and Table S2 in the supplemental material [36]. We used a randomly chosen $10 \%$ of the structures in the dataset as the test/validation data. We found that all the constructed NNPs predicted nicely both the DFT total energies and atomic forces. The RMSE values are comparable to or even better than the previous studies [15,24,27,40,46,47]. Note that the good prediction performance of the NNP with a cutoff distance of $5 \AA$ suggests the insignificance of longrange interaction in $\mathrm{Au}-\mathrm{Li}$ systems. Considering the tradeoff between the reliability of longer cutoff distances and computational costs, we use the $7 \AA$ cutoff distance and [66-10-10-1] network structure hereafter. The RMSE of total energies and atomic forces in this setting were $1.53 \mathrm{meV} /$ atom and 23.1 $\mathrm{meV} / \AA$ for the training dataset and $1.46 \mathrm{meV} /$ atom and 22.9 $\mathrm{meV} / \AA$ for the test dataset, respectively.

Figure 5 shows the comparison between DFT and NNP on the total energies and atomic forces. Both total energies and atomic forces are aligned along the diagonal lines, suggesting that the constructed NNP accurately predicted all structure types considered, i.e., $\mathrm{Au}, \mathrm{Li}, \mathrm{Au}_{3} \mathrm{Li}, \mathrm{AuLi}, \mathrm{AuLi}_{3}$, and dilute$\mathrm{Au}$ alloy. In addition, the learning curves of the total energies and atomic forces are shown in Fig. S1. The mean-squareerror (MSE) values decreased monotonically for both total energies and atomic forces, which indicates that no overfitting occurred. Note that the preceding $968(\alpha=1.0, \beta=0.0)$ and $7933(\alpha=0.5, \beta=0.5)$ iterations were performed ahead of the last training curves shown in Fig. S1.

\section{B. Lattice constants and phonon properties}

To verify the accuracy of the constructed NNP, we performed the lattice constant optimization using $\mathrm{Au}$ and $\mathrm{Li}$, as well as $\mathrm{Au}_{3} \mathrm{Li}, \mathrm{AuLi}$, and $\mathrm{AuLi}_{3}$ ordered alloys. Figures 6 and S3 show the profiles of total energy as a function of

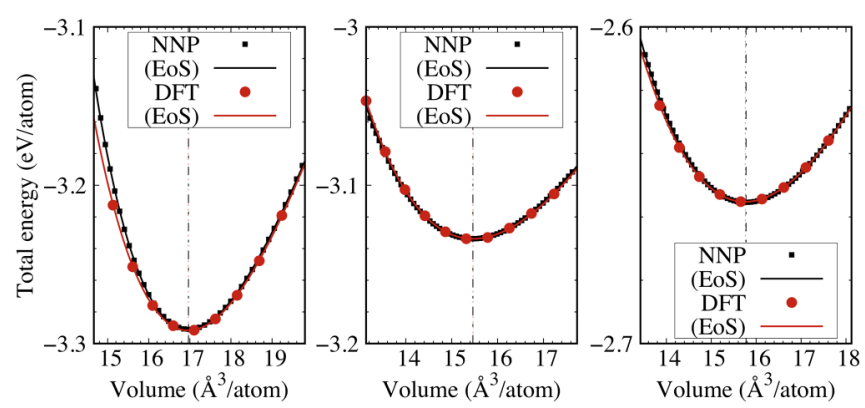

FIG. 6. Comparison of total energies as a function of volume per atom/lattice constant obtained by DFT and NNP for (left) bulk fcc $\mathrm{Au}_{3} \mathrm{Li}$, (center) bcc $\mathrm{AuLi}$, and (right) rocksalt $\mathrm{AuLi}_{3}$. The solid lines indicate the fitted curves of the equation of state. The vertical dotted line and dashed-dotted line correspond to the minimum volume per atom/lattice constant of DFT and NNP, respectively. Note that the two lines are almost overlapped.

volume per atom obtained by DFT and NNP calculations, together with the fitted Birch-Murnaghan equation of state. The vertical dashed-dotted and dotted lines correspond to the volume at the energy minima obtained by DFT and NNP, respectively. The DFT and NNP results agreed well on the minimum volume and energy: the maximum differences were smaller than $0.1 \AA^{3} /$ atom and $3.78 \mathrm{meV} /$ atom. The bulk moduli were also accurately predicted within a $10 \%$ difference, wherein the deviations mainly originated at the compressed structures. Detailed results on fitting to the Birch-Murnaghan equation are given in Sec. S3 and Table S3 in the supplemental material [36].

Next, we performed phonon calculations for fcc $\mathrm{Au}$, bcc $\mathrm{Li}$, fcc $\mathrm{Au}_{3} \mathrm{Li}$, bcc $\mathrm{AuLi}$, and rocksalt $\mathrm{AuLi}_{3}$ structures based on DFT and NNP using PHONOPY software [48]. We used the same supercell size as shown in Fig. 1 and $0.05 \AA$ displacements to calculate the force constants. Note that we used $0.02 \AA$ displacements for the $\mathrm{AuLi}_{3}$ case to circumvent the appearance of imaginary frequencies around the $\mathrm{X}$-point.

Figure 7 shows the phonon band structures and the corresponding phonon densities of states (DOSs) of fcc Au and bcc Li calculated by DFT and NNP. We found excellent agreement between the DFT- and NNP-obtained phonon modes and the peak structures of the phonon DOS. These phonon features could also be found in other studies [49-51]. Similarly, Figure 8 shows the phonon bands and the corresponding phonon DOS of fcc $\mathrm{Au}_{3} \mathrm{Li}$, bcc AuLi, and rocksalt $\mathrm{AuLi}_{3}$. The acoustic modes have been well predicted by NNP, while the optical modes of $\mathrm{Au}_{3} \mathrm{Li}$ show a slight discrepancy around the $\Gamma$-point. However, all the phonon modes match in the AuLi case. This may be attributed to the large number of $\mathrm{Au}: \mathrm{Li}=1: 1$ structures included in the training dataset. The phonon bands of $\mathrm{AuLi}_{3}$ show rather large discrepancies, which is probably due to the relatively sparse sampling of this composition (cf., Fig. $\mathrm{S} 2$, especially around the origin). Nonetheless, the phonon dispersion and energy levels were still well reproduced.

It is worth emphasizing that the phonon band structures suggest the high stability of these compounds. Note that we obtained imaginary frequencies for bcc Au and fcc Li phonon bands, which would be reasonable considering the instability of these structures (not shown). This verifies the accuracy of 

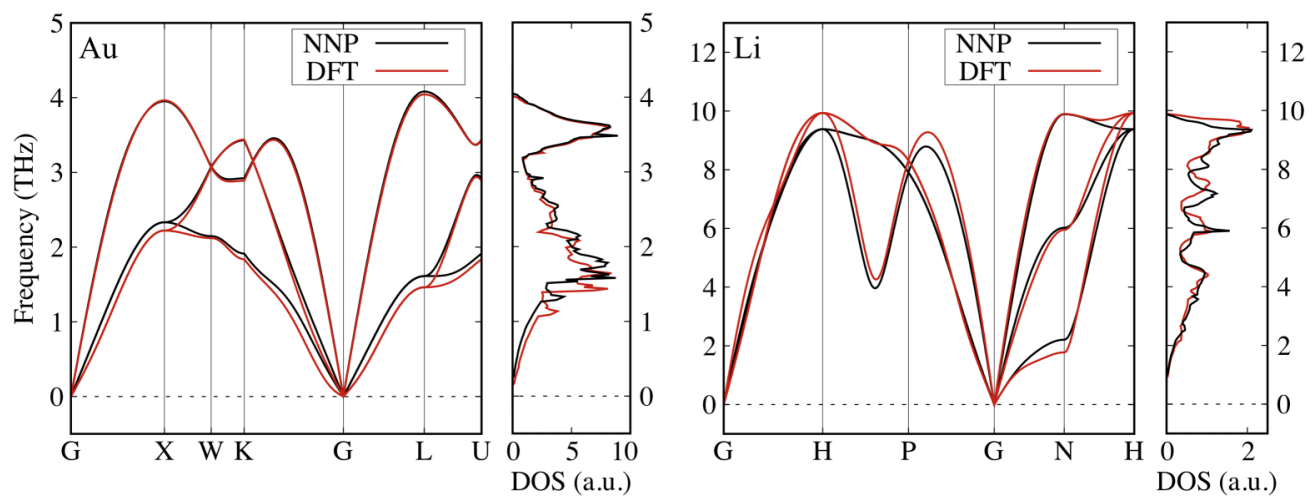

FIG. 7. Comparison of phonon bands and densities of states obtained by DFT and NNP for (left) fcc Au and (right) bcc Li.

our NNP as well as its capability to reproduce lattice parameters and phonon properties. Note that the training dataset did not include any structures used in the lattice constant optimization and phonon calculations.

\section{Phase stability of Au-Li compounds}

As mentioned in the Introduction, the stable Au- $\mathrm{Li}$ alloy phases differ among the previous research [1-4], which arises from the intrinsic complexity of miscible alloy materials and the expensive computational costs. Here, utilizing the constructed NNP, we examined the mixing energies of Au-Li with fine composition grids. To generate various structures, we used Alloy Theoretic Automated Toolkit (ATAT) software [52]. The crystal lattices used here were bcc, fcc, and hexagonalclose-packed (hcp), and these lattices had additional atoms at their octahedral (-oct) or tetrahedral (-tet) sites. In addition, rocksalt, diamond, simple cubic, $\mathrm{B} 2$, and $\mathrm{L}_{1}$ crystal structures were also taken into consideration. We fully optimized all generated structures (including lattice vectors and volume optimization) by the NNP using the conjugate gradient method with the total energy and atomic force convergence criteria of $10^{-3} \mathrm{meV}$ and $1 \mathrm{meV} / \AA$, respectively. As verification, we checked the total energies of the resulting structures using static DFT calculations. The mixing energies were calculated by $E_{\text {mix }}=E\left(\mathrm{Au}_{1-x} \mathrm{Li}_{x}\right)-\{(1-x) E(\mathrm{Au})+x E(\mathrm{Li})\}$, where $E$ is the energy per atom of the composition designated in the parentheses.

Figure 9(a) shows the calculated mixing energies obtained by the NNP. The searched points reached $89272 \mathrm{bcc}, 99711$ fcc, 86366 hcp, 43868 bcc-oct, 44333 bcc-tet, 44895 fcc-oct, 46412 fcc-tet, 46862 hcp-oct, 47413 hcp-tet, 44374 rocksalt, 45301 diamond, 46564 simple cubic, 46097 B2, and $43716 \mathrm{~L} 11$, in total 775184 structures. The minimum and maximum sizes of these structures were 2 and 24 atoms per supercell, respectively. The filled square symbols indicate the so-called convex hull points, which are connected by the solid lines. From the mixing energy profiles, we found that $\mathrm{Au}_{0.75} \mathrm{Li}_{0.25}, \mathrm{Au}_{0.5} \mathrm{Li}_{0.5}$, and $\mathrm{Au}_{0.25} \mathrm{Li}_{0.75}$ consisted of the convex hull, as is the case in the MP. In addition, we found $\mathrm{Au}_{0.625} \mathrm{Li}_{0.375}, \mathrm{Au}_{0.584} \mathrm{Li}_{0.416}, \mathrm{Au}_{0.4} \mathrm{Li}_{0.6}, \mathrm{Au}_{0.333} \mathrm{Li}_{0.667}$, $\mathrm{Au}_{0.286} \mathrm{Li}_{0.714}, \mathrm{Au}_{0.2} \mathrm{Li}_{0.8}, \mathrm{Au}_{0.111} \mathrm{Li}_{0.889}, \mathrm{Au}_{0.056} \mathrm{Li}_{0.944}$, and $\mathrm{Au}_{0.05} \mathrm{Li}_{0.95}$ as the other hull points.

Figure 9(b) shows the calculated mixing energies by DFT for the five lowest energy structures at each composition $x$, predicted by the NNP. The filled diamond symbols indicate the convex hull points among the mixing energies obtained by the DFT calculations. Here we found $\mathrm{Au}_{0.75} \mathrm{Li}_{0.25}, \mathrm{Au}_{0.625} \mathrm{Li}_{0.375}, \mathrm{Au}_{0.5} \mathrm{Li}_{0.5}, \mathrm{Au}_{0.45} \mathrm{Li}_{0.55}$, $\mathrm{Au}_{0.4} \mathrm{Li}_{0.6}, \quad \mathrm{Au}_{0.389} \mathrm{Li}_{0.611}, \quad \mathrm{Au}_{0.357} \mathrm{Li}_{0.643}, \quad \mathrm{Au}_{0.333} \mathrm{Li}_{0.667}$, $\mathrm{Au}_{0.25} \mathrm{Li}_{0.75}$, and $\mathrm{Au}_{0.2} \mathrm{Li}_{0.8}$ as the hull points. Figure 9(c) shows the convex hull points of both NNP and DFT. Although some of the hull points differed between NNP and DFT, the two convex hull lines overlapped considerably, suggesting the accuracy of the NNP predictions. Moreover, all of the NNP hull points were located in the vicinity of the DFT hull lines, where the mixing energy difference was $\sim 6.31$ $\mathrm{meV} /$ atom $(x=0.375)$ at most. In addition to the $12(10)$ NNP (DFT) convex hull points, we also found a large number of points located slightly above the hull over the entire
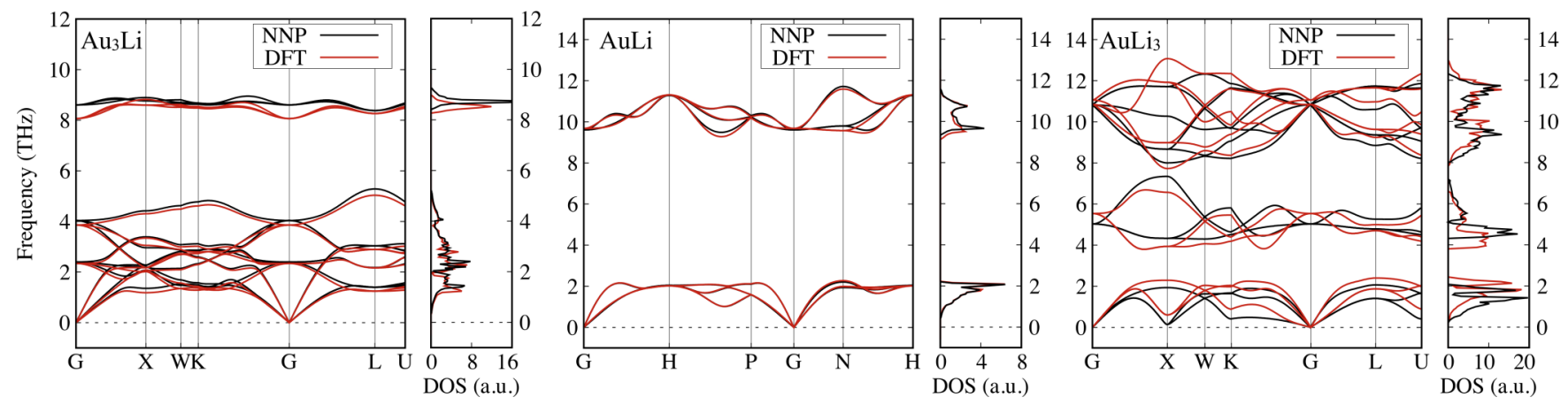

FIG. 8. Comparison of phonon bands and densities of states obtained by DFT and NNP for (left) fcc Au 3 Li, (center) bcc AuLi, and (right) rocksalt $\mathrm{AuLi}_{3}$ ordered alloys. 

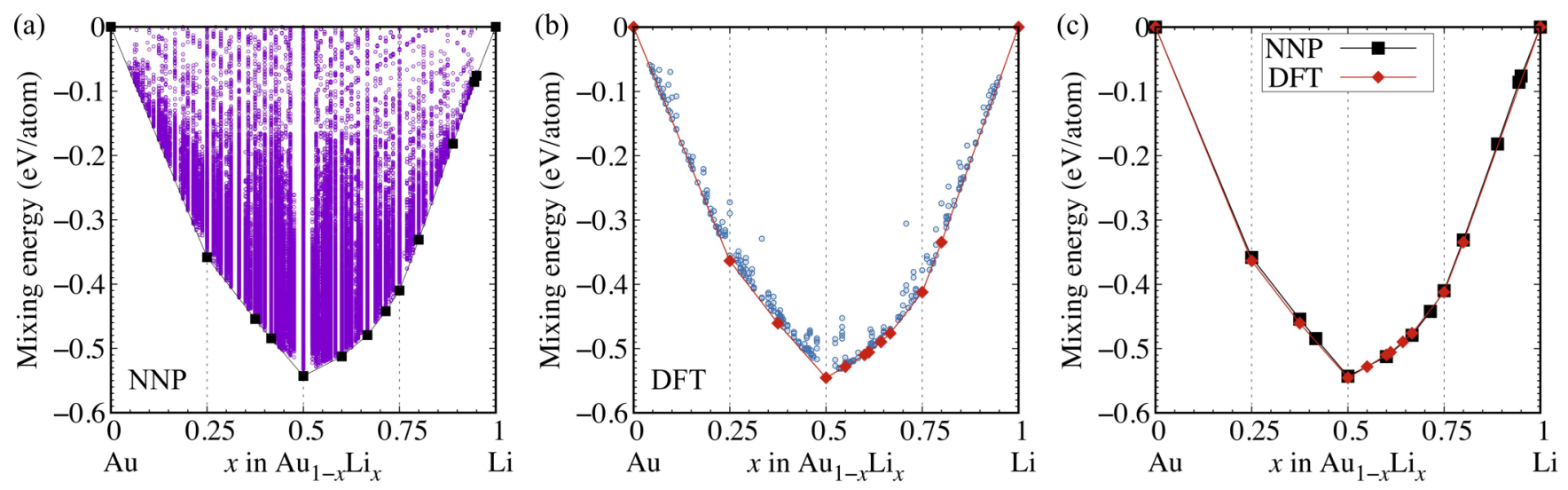

FIG. 9. (a) Calculated mixing energy of $\mathrm{Au}_{1-x} \mathrm{Li}_{x}$ using NNP. Filled squares show the convex hull points, which are connected by the solid lines. (b) Calculated mixing energies by DFT for the five lowest energy structures at each composition $x$, predicted by NNP. Filled diamonds show the convex hull points, which are connected by the solid lines. (c) Comparison of the convex hull points between NNP and DFT.

compositional space. These compositions may appear in a specific experimental condition as metastable phases.

Figure 10 shows the structural images corresponding to the DFT convex hull points of $x=0.55,0.611$, and 0.643 . The space groups of the structures at $x=0.55,0.611$, and 0.643 are $C m, P 3 m 1$, and $A m m 2$, respectively. In addition, the common neighbor analysis method [53] identified that $x=0.611$ has bec crystal structure. The structure information of the other convex hull points is shown in Fig. S4 in the supplemental material [36].

We also performed mixing energy calculations for the previously reported stable phases, i.e., $\mathrm{Au}_{2} \mathrm{Li}(x=0.333)$, $\mathrm{Au}_{3} \mathrm{Li}_{5} \quad(x=0.625)$, and $\mathrm{Au}_{4} \mathrm{Li}_{15} \quad(x=0.789)$, starting from the provided space group and structure information $[2,4,10,11]$. As a result, we found that the mixing energies of $x=0.333$ and 0.625 are located slightly higher (within $10 \mathrm{meV} /$ atom) than the hull lines shown in Fig. 9. However, $x=0.789$ had a $4.34 \mathrm{meV} /$ atom lower value than the DFT hull lines (please refer to Sec. S4 in the supplemental material for details [36]). Note that the $\mathrm{Au}_{4} \mathrm{Li}_{15}$ phase seen in MP consisting of 76 atoms per supercell was out of our search range. We expect that the inclusion of $x=0.789$ will replace the hull point of $x=0.8$. The subtle differences in mixing energies in the order of a few $\mathrm{meV} /$ atom could be easily buried by circumstances and/or numerical accuracy. Thus, observing a part of the original phase is unavoidable. The discrepancy among previous studies mentioned earlier can be understood from the above reasoning.
We next carried out NNP-MD simulations using $\mathrm{Au}_{3} \mathrm{Li}$, AuLi, $\mathrm{AuLi}_{3}$, and $\mathrm{Au}_{4} \mathrm{Li}_{15}$ as the base structures (cf., Fig. 1 and Fig. S6 in the supplemental material [36]), and $\mathrm{Au}$ and $\mathrm{Li}$ atoms were randomly replaced for the sake of compositional variations. The resulting mixing energy profile at $T=500 \mathrm{~K}$ revealed that the convex hull points were a little different from those at $T=0 \mathrm{~K}$ (please refer to Fig. S7 in the supplemental material for details [36]). This again indicates that observed phases would be susceptible to experimental conditions.

From the $N V T$-MD trajectories, we computed the statistically averaged mean-square displacement of atoms. We then evaluated the diffusion coefficients of various $\mathrm{Au}_{1-x} \mathrm{Li}_{x}$ compositions using the Einstein relation. We found that the diffusion coefficient tends to become larger with the composition $x$ (please refer to Fig. S8 in the supplemental material for details [36]). This may suggest that an accumulation of excess $\mathrm{Li}$ on the $\mathrm{Au}$ electrode will facilitate the alloying process. However, a detailed analysis seems necessary to confirm this, because correlation between the composition and diffusion coefficient is not very high.

As demonstrated above, our constructed NNP can be used over the entire range of binary alloy compositions, despite our training dataset including only a limited number of $\mathrm{Au}-\mathrm{Li}$ ratios, $x$. The precise investigation of mixing energy reveals the compositions that were not present in the previous studies. For further detailed analyses, generation of larger sized structures is desirable, which involves a considerable increase in the number of structures at each $x$ as well as compositional (a) $x=0.55\left(\mathrm{Au}_{9} \mathrm{Li}_{11}\right)$

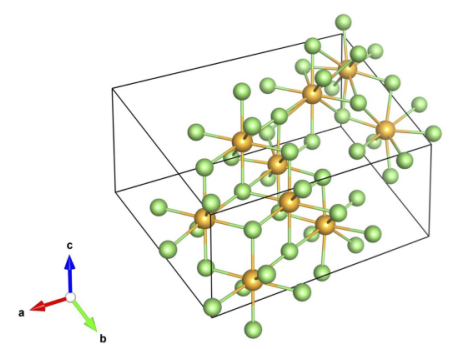

(b) $x=0.611\left(\mathrm{Au}_{7} \mathrm{Li}_{11}\right)$

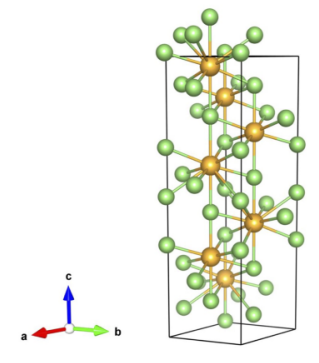

(c) $x=0.643\left(\mathrm{Au}_{5} \mathrm{Li}_{9}\right)$

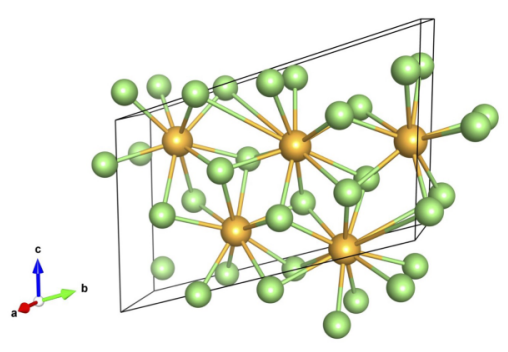

FIG. 10. Structural images of (a) $\mathrm{Au}_{0.45} \mathrm{Li}_{0.55}$, (b) $\mathrm{Au}_{0.389} \mathrm{Li}_{0.611}$, and (c) $\mathrm{Au}_{0.357} \mathrm{Li}_{0.643}$, which correspond to the convex hull points. 

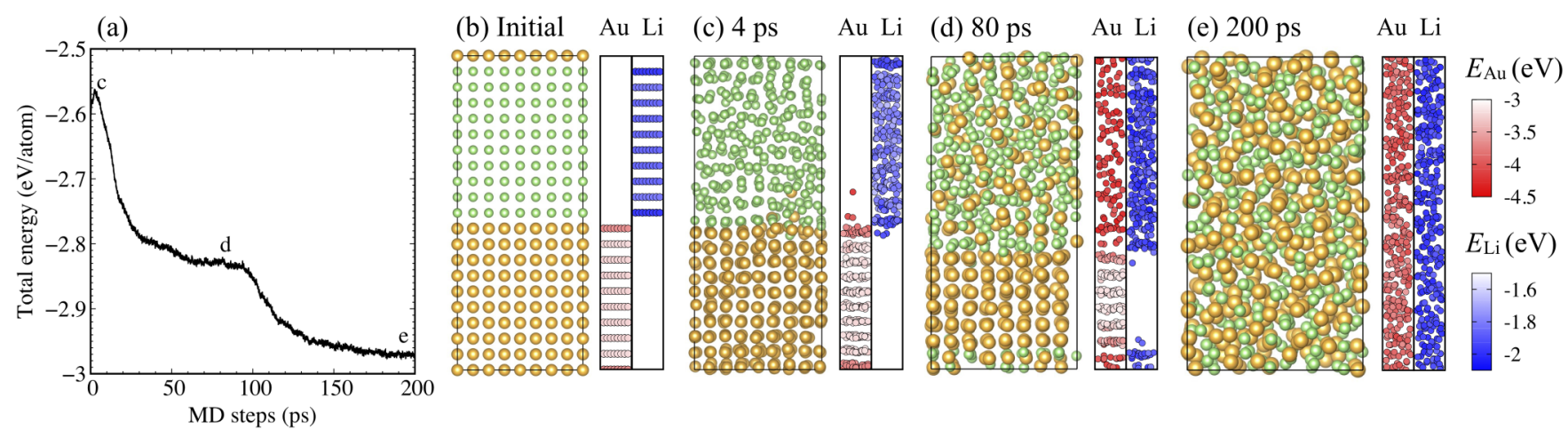

FIG. 11. (a) Total energy profile of the NNP-MD simulation, and its snapshots of (b) initial, (c) 4 ps, (d) 80 ps, and (e) 200 ps. The atomic energies of $\mathrm{Au}$ and $\mathrm{Li}$ are separately shown in the right-hand side of each snapshot using relative atomic positions. The colors correspond to the magnitude of the atomic energies.

variations. A more efficient method and/or code than ATAT seems necessary to do this. We also note that the present NNP would enable us to draw a reasonable phase diagram (e.g., temperature versus composition), considering the phonon prediction capability. We shall leave these issues as future tasks.

Next, to clarify the atomic-scale picture of the alloying process, we carried out NNP-MD (NPT at $T=500 \mathrm{~K}$ and $P=0 \mathrm{GPa})$ simulations starting from the phase-separated structure, consisting of 10 atomic layers (32 atoms/layer) of fcc Au and Li using the Au lattice constant [Fig. 11(b)]. Figure 11(a) shows the total energy profile along the simulation time, and Figs. 11(b)-11(e) show the MD snapshots together with the atomic energy profiles. The colors indicate the magnitude of atomic energies along with the relative atomic positions.

We found that at $4 \mathrm{ps}$, a single Au atom dissolved into the bulk Li [Fig. 11(c)]. This occurred due to the slightly larger energy gain of Au dissolving into $\mathrm{Li}$ than the Li dissolving into $\mathrm{Au}$, which is corroborated by the mixing energies (cf., Fig. 9). This was followed by continued Au alloying into the Li until around $80 \mathrm{ps}$, where the total energy reached a plateau [Fig. 11(a)]. At this plateau, Li mixed with Au over the entire Li bulk region, while six layers of Au remained intact [Fig. 11(d)]. The tendency for a larger composition $x$ to have a higher diffusion coefficient (cf., Fig. S8) matches with this observation. The cell volume gradually decreased by reducing the length of the $c$-axis, which is attributed to the smaller optimal volume of alloys (cf., Figs. 6 and S3). The alloying was reinitiated at $\sim 100 \mathrm{ps}$ with the emergence of a second plateau in the total energy profile. At $200 \mathrm{ps}$, complete alloying and equilibration [cf., Fig. 11(e)] was reached.

The solution of $\mathrm{Au}$ atoms into $\mathrm{Li}$ at the initial stage of alloying is a probabilistic process, and it proceeds independently at both $\mathrm{Au}-\mathrm{Li}$ interfaces. When multiple adjacent $\mathrm{Au}$ atoms dissolved into $\mathrm{Li}$, the alloying of the entire interface started from the dissolved region. This corresponds to the steep energy decrease from $\sim 4$ to $30 \mathrm{ps}$ as shown in Fig. 11(a). In the present case, the dissolution of the lower interface initially took place. The upper interface follows from the inflection point of the energy profile at $\sim 12$ ps. Note that the initial alloying process using the model consisting of 10 atomic layers of fcc $\mathrm{Au}$ and $\mathrm{Li}$ with 128 atoms/layer is shown in Sec. S5 in the Supplemental Material [36].
In terms of atomic energies, atoms located at the interface had lower values (Au: $-3.71 \mathrm{eV}, \mathrm{Li}:-2.02 \mathrm{eV}$ ) compared to those of the bulk region (Au: $-3.28 \mathrm{eV}, \mathrm{Li}:-1.89 \mathrm{eV})$ at the initial structure. Atoms at the second layer from the interface, conversely, had higher values ( $\mathrm{Au}:-3.23 \mathrm{eV}, \mathrm{Li}:-1.84 \mathrm{eV}$ ). This shows that the Au-Li sharp interface will spontaneously form an alloy. While thermal oscillation caused the atomic energy fluctuation at the bulk region, e.g., $41.0 \mathrm{meV}$ at $4 \mathrm{ps}$ and $51.3 \mathrm{meV}$ at $80 \mathrm{ps}$, the interface atoms had much lower energies. Once $\mathrm{Au}$ atoms mixed with $\mathrm{Li}$ atoms, the atomic energies decreased further. A major part of the atomic energy change by alloying was absorbed into the Au section. We shall also leave this point for future work.

As demonstrated above, due to the high stability of the $\mathrm{Au}-\mathrm{Li}$ system, $\mathrm{Au}$ and $\mathrm{Li}$ atoms spontaneously mixed in the simulations within a few hundred picoseconds, which is a noticeably short period compared to the operating time of ionic devices. This alloying property, in other aspects, leads to the stabilization of interfaces of secondary batteries using a Li anode (cf., e.g., Refs. [6,7]). On the other hand, controlling the amount of $\mathrm{Li}$ introduced into $\mathrm{Au}$ must be a significant factor for developing devices using ion conduction, such as VolRAM [8].

\section{CONCLUSIONS}

We constructed a neural network potential (NNP) for $\mathrm{Au}-$ Li binary systems based on density functional theory (DFT) calculations. We used a total of 9285 structures, including pure $\mathrm{Au}$ and $\mathrm{Li}$, ordered $\mathrm{Au}_{3} \mathrm{Li}, \mathrm{AuLi}$, and $\mathrm{AuLi}_{3}$, dilute $\mathrm{Au}$ alloys, and $\mathrm{Au} / \mathrm{Li}$ superlattices as our dataset. We efficiently generated these structures using NNP-based MD simulations, where unique structures were identified by principal component analysis based on symmetry functions. The predicted lattice parameters and phonon properties obtained by the NNP agree well with those from DFT calculations. We also investigated the mixing energy of $\mathrm{Au}_{1-x} \mathrm{Li}_{x}$, showing consistency with the DFT results over a wide range of compositional space. We successfully reproduced the convex hull points of $\mathrm{Au}_{3} \mathrm{Li}(x=0.25), \mathrm{Au}_{5} \mathrm{Li}_{3}(x=0.375)$, AuLi $(x=$ $0.5), \mathrm{Au}_{2} \mathrm{Li}_{3}(x=0.6), \mathrm{AuLi}_{2}(x=0.667)$, and $\mathrm{AuLi}_{3}(x=$ 0.75 ), which were reported previously. Additionally, we found three new alloy compositions of $\mathrm{Au}_{9} \mathrm{Li}_{11}(x=0.55), \mathrm{Au}_{7} \mathrm{Li}_{11}$ 
$(x=0.611)$, and $\mathrm{Au}_{5} \mathrm{Li}_{9}(x=0.643)$, consisting of the convex hull obtained by the DFT verifications. We further verified that the previously reported phases of $\mathrm{Au}_{2} \mathrm{Li}(x=0.333)$ and $\mathrm{Au}_{3} \mathrm{Li}_{5}(x=0.625)$ are located slightly higher than the convex hull, while $\mathrm{Au}_{4} \mathrm{Li}_{15}(x=0.789)$ exhibited the lower energy. For compositions where there are discrepancies in stability among previous studies, we found that a small energy change of several $\mathrm{meV} /$ atom can alter whether or not they become stable phases. This is considered to be the origin of the discrepancies. Considering a vast search space and marginal energy differences among alloy compositions, inexpensive and accurate simulations using machine learning interatomic potentials could be utilized for further studies.

Finally, we examined the alloying process starting from the phase-separated structure to the complete mixing phase using our relatively large system. We found that at the initial stage of alloying, a single Au atom tends to dissolve into the $\mathrm{Li}$ region. Furthermore, we determined that when multiple adjacent $\mathrm{Au}$ atoms dissolve into $\mathrm{Li}$, the alloying of the entire $\mathrm{Au} / \mathrm{Li}$ interface starts from the dissolved region.
In this study, we successfully constructed an accurate NNP via the proposed workflow. This would help to accelerate applications of ML interatomic potentials toward more complicated systems.

The NNP program and the LAMMPS interface developed in this work are available in the GitHub site [54]. The DFT dataset used for the NNP constructions is stored there as well.

\section{ACKNOWLEDGMENTS}

This work was supported by the JST CREST Program "Novel electronic devices based on nanospaces near interfaces" and JSPS KAKENHI Grants No. 19H02544, No. 19K15397, No. 20K15013, and No. 20H05285. Some of the calculations presented here were performed using the computer facilities at ISSP Supercomputer Center and Information Technology Center, The University of Tokyo, and Institute for Materials Research, Tohoku University (MASAMUNEIMR). We would like to thank Editage [55] for English language editing.
[1] A. D. Pelton, Bull. Alloy Phase Diagrams 7, 228 (1986).

[2] P. Bach, I. Valencia-Jaime, U. Rütt, O. Gutowski, A. H. Romero, and F. U. Renner, Chem. Mater. 28, 2941 (2016).

[3] P. Bach, M. Stratmann, I. Valencia-Jaime, A. H. Romero, and F. U. Renner, Electrochem. Acta 164, 81 (2015).

[4] G. Yang, Y. Wang, F. Peng, A. Bergara, and Y. Ma, J. Am. Chem. Soc. 138, 4046 (2016).

[5] Z. Zeng, W.-I. Liang, Y.-H. Chu, and H. Zheng, Faraday Discuss. 176, 95 (2014).

[6] A. Kato, A. Hayashi, and M. Tatsumisago, J. Power Sources 309, 27 (2016).

[7] A. Kato, H. Kowada, M. Deguchi, C. Hotehama, and A. Hayashi, Solid State Ion. 322, 1 (2018).

[8] I. Sugiyama, R. Shimizu, T. Suzuki, K. Yamamoto, H. Kawasoko, S. Shiraki, and T. Hitosugi, APL Mater. 5, 046105 (2017).

[9] K. Shimizu, W. Liu, W. Li, S. Kasamatsu, Y. Ando, E. Minamitani, and S. Watanabe, Phys. Rev. Mater. 4, 015402 (2020).

[10] http://www.materialsproject.org.

[11] A. Jain, S. P. Ong, G. Hautier, W. Chen, W. D. Richards, S. Dacek, S. Cholia, D. Gunter, D. Skinner, G. Ceder, and K. A. Persson, APL Mater. 1, 011002 (2013).

[12] J. Luytena, J. D. Keyzer, P. Wollants, and C. Creemers, Calphad 33, 370 (2009).

[13] M. Ångqvist, J. M. Rahm, L. Gharaee, and P. Erhart, Phys. Rev. Materials 3, 073605 (2019).

[14] S. M. Aspera, R. L. Arevalo, K. Shimizu, R. Kishida, K. Kojima, N. H. Linh, H. Nakanishi, and H. Kasai, J. Electron. Mater. 46, 3776 (2017).

[15] J. Behler and M. Parrinello, Phys. Rev. Lett. 98, 146401 (2007).

[16] A. P. Bartók, M. C. Payne, R. Kondor, and G. Csányi, Phys. Rev. Lett. 104, 136403 (2010).

[17] A. P. Thompson, L. P. Swiler, C. R. Trott, S. M. Foiles, and G. J. Tucker, J. Compt. Phys. 285, 316 (2015).
[18] G. C. Sosso, G. Miceli, S. Caravati, J. Behler, and M. Bernasconi, Phys. Rev. B 85, 174103 (2012).

[19] N. Artrith and A. M. Kolpak, Comput. Mater. Sci. 110, 20 (2015).

[20] S. Chiriki, S. Jindal, and S. S. Bulusu, J. Chem. Phys. 147, 154303 (2017).

[21] S. Hajinazar, J. Shao, and A. N. Kolmogorov, Phys. Rev. B 95, 014114 (2017).

[22] R. Kobayashi, D. Giofré, T. Junge, M. Ceriotti, and W. A. Curtin, Phys. Rev. Materials 1, 053604 (2017).

[23] N. Artrith, A. Urban, and G. Ceder, J. Chem. Phys. 148, 241711 (2018).

[24] B. Onat, E. D. Cubuk, B. D. Malone, and E. Kaxiras, Phys. Rev. B 97, 094106 (2018).

[25] T. Wen, C.-Z. Wang, M. J. Kramer, Y. Sun, B. Ye, H. Wang, X. Liu, C. Zhang, F. Zhang, K.-M. Ho, and N. Wang, Phys. Rev. B 100, 174101 (2019).

[26] X.-G. Li, C. Hu, C. Chen, Z. Deng, J. Luo, and S. P. Ong, Phys. Rev. B 98, 094104 (2018).

[27] W. Li, Y. Ando, E. Minamitani, and S. Watanabe, J. Chem. Phys. 147, 214106 (2017).

[28] A. Marcolongo, T. Binninger, F. Zipoli, and T. Laino, Chem. Syst. Chem. 2, e1900031 (2019).

[29] S. Fujikake, V. L. Deringer, T. H. Lee, M. Krynski, S. R. Elliott, and G. Csányi, J. Chem. Phys. 148, 241714 (2018).

[30] K. Momma and F. Izumi, J. Appl. Cryst. 44, 1272 (2011).

[31] J. P. Perdew, K. Burke, and M. Ernzerhof, Phys. Rev. Lett. 77, 3865 (1996).

[32] P. E. Blöchl, Phys. Rev. B 50, 17953 (1994).

[33] H. J. Monkhorst and J. D. Pack, Phys. Rev. B 13, 5188 (1976).

[34] G. Kresse and J. Furthmüller, Comput. Mater. Sci. 6, 15 (1996).

[35] G. Kresse and J. Furthmüller, Phys. Rev. B 54, 11169 (1996).

[36] See Supplemental material at http://link.aps.org/supplemental/ 10.1103/PhysRevB.103.094112 for additional computational settings of NNPs, and additional calculations and analyses of $\mathrm{Au}-\mathrm{Li}$ systems. 
[37] J. L. Morales and J. Nocedal, ACM Trans. Math. Softw. 38, 7 (2011).

[38] S. Plimpton, J. Comp. Phys. 117, 1 (1995).

[39] LAMMPS software, https://lammps.sandia.gov/.

[40] W. Jeong, K. Lee, D. Yoo, D. Lee, and S. Han, J. Phys. Chem. C 122, 22790 (2018).

[41] K. Lee, D. Yoo, W. Jeong, and S. Han, Compt. Phys. Commun. 242, 95 (2019).

[42] E. D. Cubuk, B. D. Malone, B. Onat, A. Waterland, and E. Kaxiras, J. Chem. Phys. 147, 024104 (2017).

[43] N. Artrith and J. Behler, Phys. Rev. B 85, 045439 (2012).

[44] W. Li and Y. Ando, J. Chem. Phys. 151, 114101 (2019).

[45] G. Sivaraman, A. N. Krishnamoorthy, M. Baur, C. Holm, M. Stan, G. Csányi, C. Benmore, and Á. Vázquez-Mayagoitia, npj Comput. Mater. 6, 104 (2020).
[46] E. Minamitani, M. Ogura, and S. Watanabe, Appl. Phys. Express 12, 095001 (2019).

[47] N. Artrith, T. Morawietz, and J. Behler, Phys. Rev. B 83, 153101 (2011).

[48] A. Togo and I. Tanaka, Scr. Mater. 108, 1 (2015).

[49] J. W. Lynn, H. G. Smith, and R. M. Nicklow, Phys. Rev. B 8, 3493 (1973).

[50] A. D. Corso, J. Phys.: Condens. Matter 25, 145401 (2013).

[51] B. Xu and M. J. Verstraete, Phys. Rev. Lett. 112, 196603 (2014).

[52] A. van de Walle and G. Ceder, J. Phase Eq. 23, 348 (2002).

[53] A. Stukowski, Model. Sumul. Mater. Sci. Eng. 20, 045021 (2012).

[54] https://github.com/shimizu00000/nnp.git.

[55] www.editage.com. 